\title{
IMPLICIT LEARNING: A SYMPOSIUM
}

\section{Introduction}

A survey of the titles of articles across a wide spectrum of journals will indicate that many researchers are currently studying implicit processes and processing. We see numerous studies of implicit learning, implicit memory, subliminal perception, and other varieties of implicit cognition. The definitions of implicit vary somewhat across the domains of research and form a fuzzy set. The usual contrast is made between implicit processes, which are thought to occur without much volition or conscious attention and whose operation is difficult to capture verbally, and other (explicit) processes, which require conscious attention and permit greater verbal description. Many experiments have been done in the attempt to answer whether explicit and implicit processes differ qualitatively - obey different laws-or rather are simply end points on a continuum (i.e., processes that vary quantitatively but that are not fundamentally different).

This issue of the journal presents a symposium on the topic of implicit learning, the origins of which can be traced back to early work by Thorndike and Hull, among others. The spark that ignited contemporary interest in implicit learning, however, can probably be traced to studies begun in the 1960s by Arthur Reber, on implicit learning of artificial grammars. The issues he (and then others) raised have been refined and advanced in many other domains and paradigms, so that a voluminous literature exists on these topics. Critical examination of this body of research is timely.

Origins of this symposium were serendipitous. The two lead articles, by Dienes and Berry and by Neal and Hesketh, were submitted independently by researchers from two different continents to the journal office (on yet a third continent). Both wended their separate ways through the review process, were revised, and, by pure happenstance, landed on my desk at the same time for final editorial decisions. I found myself with two literature reviews on roughly the same body of work, but ones that took different perspectives, had different concerns, and in some cases reached different conclusions. Both were excellent papers and were accepted. I decided to make this somewhat unusual occurrence the occasion for this symposium. Reviewers of the two manuscripts had written persuasive essays agreeing and disagreeing with various points raised in the papers. Some of these reviewers were invited to participate in the symposium by providing commentaries on the main papers. These comments were themselves reviewed and revised, and then the authors of the two target papers replied to these comments.

The symposium ranges widely across the study of implicit learning, calling attention to several thorny issues and advancing discussion and thought on these topics. I hope that readers will enjoy these fine contributions and learn much from them. 